\title{
Isolation Improvement Technique for Two Closely Spaced Loop Antennas Using MTM Absorber Cells
}

\author{
Hongmin Lee and Hyungsup Lee \\ Department of Electronic Engineering, Kyonggi University, Suwon, Kyonggi-do 443-760, Republic of Korea \\ Correspondence should be addressed to Hongmin Lee, hmlee@kyonggi.ac.kr
}

Received 19 December 2011; Revised 3 March 2012; Accepted 5 March 2012

Academic Editor: Mandeep Jit Singh

Copyright () 2012 H. Lee and H. Lee. This is an open access article distributed under the Creative Commons Attribution License, which permits unrestricted use, distribution, and reproduction in any medium, provided the original work is properly cited.

This paper presents a new method to improve isolation between two loop antennas with absorber cells exhibiting negative permittivity and permeability at the aimed frequency of $2.54 \mathrm{GHz}$. The metamaterial (MTM) unit cell was constructed by two open complementary split ring resonator (OCSRR) and a split ring resonator (SRR) arrangement. The proposed antenna structure consists of two loop antennas occupying a space with the dimensions of $51 \mathrm{~mm} \times 24 \mathrm{~mm} \times 2 \mathrm{~mm}$ and three absorber cells. The size of each absorber unit cell is $7.4 \mathrm{~mm} \times 7 \mathrm{~mm} \times 2 \mathrm{~mm}$. The isolation of more than $20 \mathrm{~dB}$ was achieved with three absorber cells loaded between two loop antennas; interantenna spacing (center to center) is $0.23 \lambda_{0}$ at $2.54 \mathrm{GHz}$. The results show that port-to-port isolation between two antennas can be improved with absorber.

\section{Introduction}

The increased demand for higher data rates and high-quality service in wireless communication is driving the researcher to investigate new ways to operate multiple antennas. In order to increase the communication performance, utilization of multiple input multiple output (MIMO) antenna systems has been regarded as one of practical approaches [1]. Whereas the use of multiple antennas in MIMO systems improves the performance and channel capacity of wireless communication systems, the physical limitations inherent in wireless devices pose some challenges. It seems obvious that the antenna elements in such a small wireless device would need to be closely spaced, which would result in a significant amount of mutual coupling between the antennas. The mutual coupling between antennas reduces their efficiencies as part of their power would generally be radiated into the other antenna. This coupling may also cause unwanted interference between them. Therefore, isolation improvement techniques between antennas have become a very important topic. A standard method in improving isolation is to limit the radiation in the propagating direction and to increase the distance between the antennas. However, this method is not highly efficient because the area allocated to the antennas in mobile device is very small. In order to improve the isolation performance, several studies have been done on spatial diversity and MIMO systems. In [2,3], multiple slits etched on a single ground plane were used to isolate the feeding port of two closely packed antennas. Another technology is presented in $[4,5]$, where two antennas are linked with a so-called neutralization line in order to increase the portto-port isolation. However, the aforementioned techniques are only suitable for mobile diversity and MIMO antennas with a common ground plane. This study presents a new method to improve the isolation between two antennas without a shared ground plane. In order to increase the isolation between the two loop antennas, double negative metamaterial (MTM) absorber unit cells were used. The metamaterials (MTMs) are artificial composite structures that exhibit a homogeneous effective permittivity $\varepsilon$ and permeability $\mu$, which become negative over an operating frequency range [6]. Due to the unique characteristics of the MTMs and their integration, a number of the potential MTM applications have been researched in many small antenna applications. A MTM absorber consists of a structure with negative permittivity and another structure with negative permeability, resulting in a negative refractive index [7]. In this paper, we use a double-layered structure with a SRR and two open complementary split ring resonators (OCSRRs) [8] which are put on top of each other in order to make a miniaturized thin MTM absorber unit cell for the $2 \mathrm{GHz}$ frequency band. In the design of the proposed antenna 


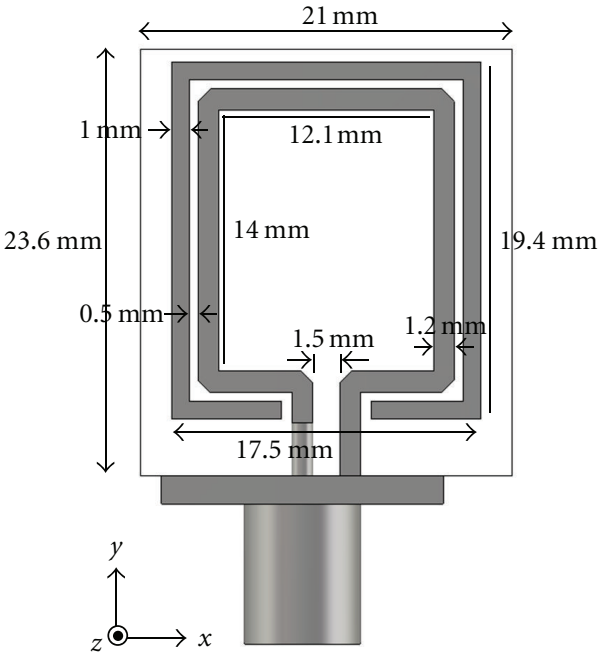

(a)

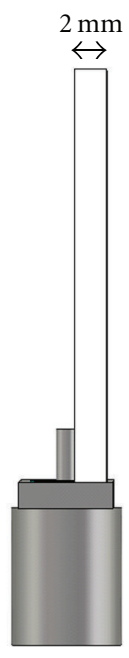

(b)

Figure 1: Geometry of the proposed loop antenna: (a) top view; (b) side view.

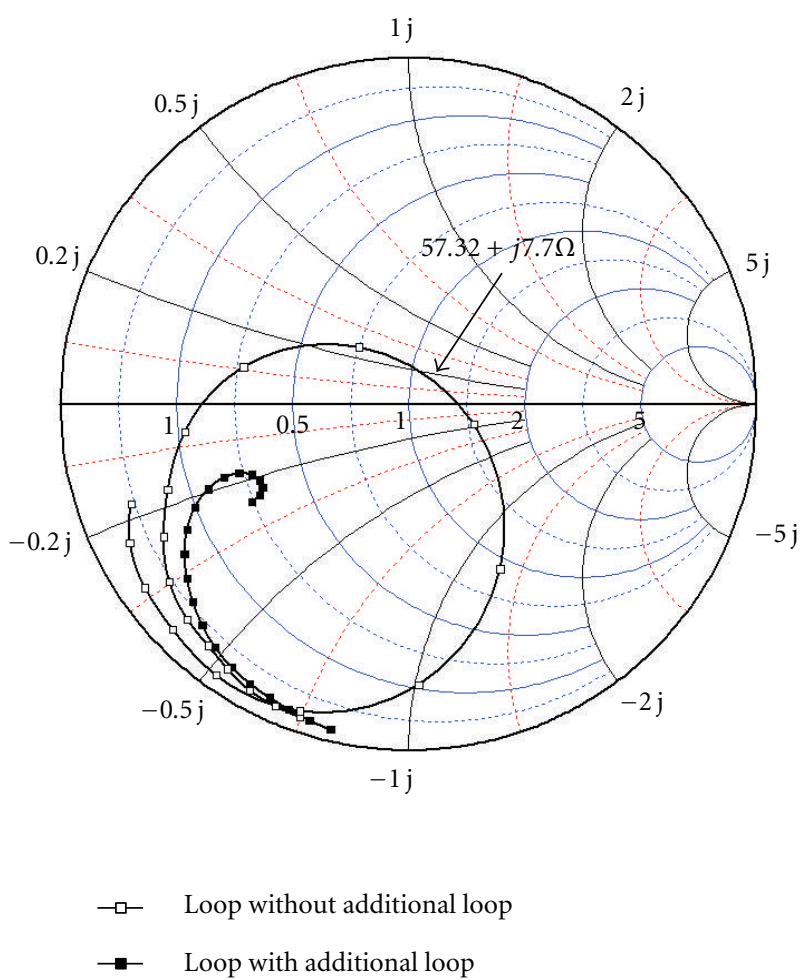

FIGURE 2: Simulated input impedance from $2 \mathrm{GHz}$ to $3 \mathrm{GHz}$ for the proposed loop antenna with/without additional loop strip.

employing MTM absorber cells, the $3 \mathrm{D}$ field simulation tool CST MWS (microwave studio) was used.

\section{The Design of a Loop Antenna and MTM Absorber Unit Cell}

The geometry of the proposed loop antenna is shown in Figure 1 . It is fed by a $50 \Omega$ coaxial connector and designed on a FR-4 dielectric substrate (relative permittivity $=4.6$, thickness $=2 \mathrm{~mm}$ ). The proposed antenna consists of a small rectangular loop and an additional split ring resonator (SRR) shape loop strip. The single loop antenna occupied $21 \mathrm{~mm} \times 23.6 \mathrm{~mm} \times 2 \mathrm{~mm}$; the total length of the inner loop is $54 \mathrm{~mm}$ long $\left(\approx \lambda_{g}\right.$ at $\left.2.5 \mathrm{GHz}\right)$.

An electrically small loop antenna exhibits very small resistance, and its reactance is inductive below its self-resonance point. However, the input impedance of a resonant loop antenna varies with circumference length of the loop 


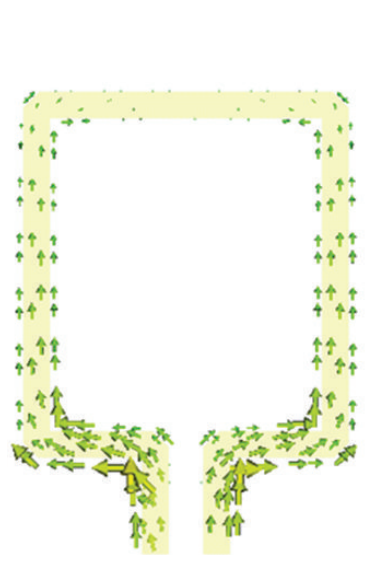

(a)

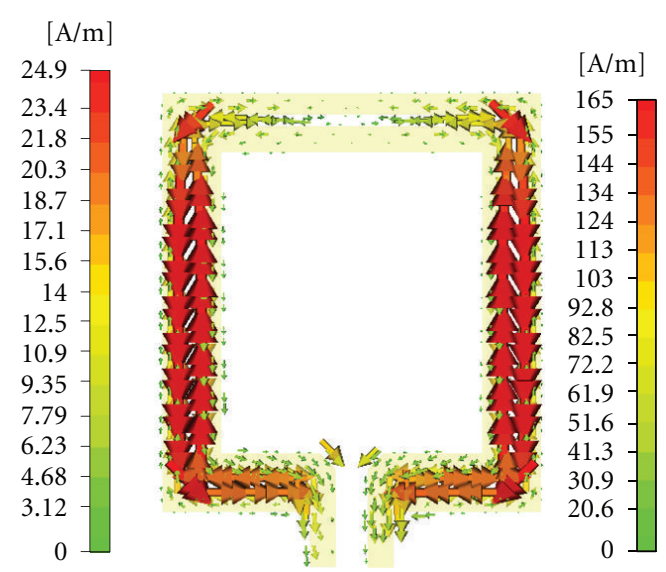

(b)

FIGURE 3: Comparison of the simulated surface current distributions: (a) a rectangular loop; (b) a rectangular loop with an additional loop strip.

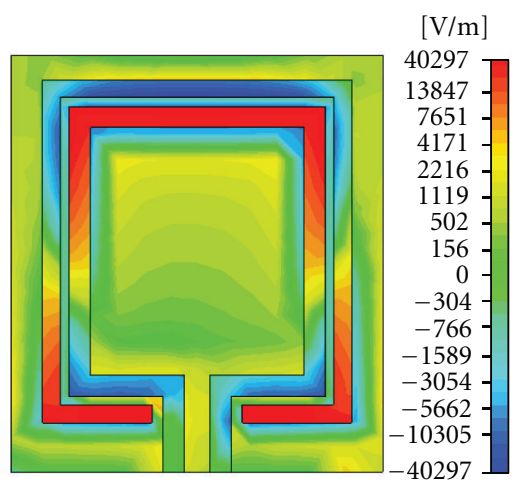

(a)
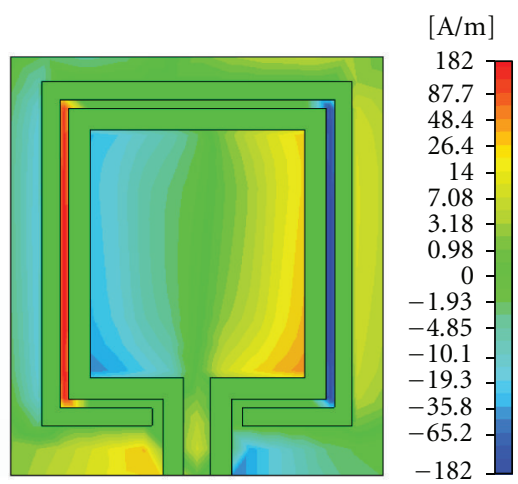

(b)

FIGURE 4: Simulated electric and magnetic field distributions of the proposed loop antenna: (a) electric field; (b) magnetic field.

conductor. As the electrical size of the loop antenna is increased, the reactance becomes zero at antiresonant point (one-half wavelength loop antenna) and resonant point (one wavelength loop antenna). Figure 2 shows a smith chart that plots calculated characteristics of input impedance for the proposed loop antenna with/without an additional loop strip, where the span of frequency is from $2 \mathrm{GHz}$ to $3 \mathrm{GHz}$. As can be seen in the figure, a loop antenna without an additional loop strip acts as a capacitive element. When the additional loop strip is used, the value of input impedance at the aimed frequency $(2.54 \mathrm{GHz})$ is about $57.3+j 7.7 \Omega$. Hence, the additional loop strip acts as an inductive element for impedance matching.

A comparison of the simulated surface current distributions for the proposed loop antennas with/without an additional loop structure are shown in Figure 3. In Figure 3(a), the currents on the two vertical loop arms flow in the same direction and have a null in middle of the top loop section. The currents are seen to be equivalent to that of a pair of parallel monopole antennas driven in-phase with a spacing of approximately $1 / 8 \lambda_{g}$. In the simulation result, as shown in Figure 3(a), light green color indicates smaller surface current flow on the loop strips due to the input impedance mismatch. When the additional loop strip structure is added, there are two circulating current loops on both sides of two vertical loops and additional loop strip arms, as shown in Figure 3(b). As a result, the current distribution is seen to be roughly equivalent to that a pair of parallel loop antenna driven in out-of-phase and with a spacing approximately equal to the diameter of the inner loop. Figure 4 presents the simulated electric field and magnetic field distributions of the proposed antenna. As observed in the Figure 4(a), red color indicates large positive values and blue color indicates large negative values for the electric field. It means that the field direction opposite to the loop conductor axes. A capacitance is produced between the inner and outer loop strips and this forms circular loop current, as shown in Figure 3(b). As a result, the magnetic field is concentrated on the gaps of two vertical loop's arms, as shown in Figure 4(b).

According to the effective medium theory, MTMs can be represented by the complex values of electric permittivity $\varepsilon_{\text {eff }}$ $\left(=\varepsilon^{\prime}+j \varepsilon^{\prime \prime}\right)$ and magnetic permeability $\mu_{\text {eff }}\left(=\mu^{\prime}+j \mu^{\prime \prime}\right)$. By varying the dimensions of the electric and magnetic components, it is possible to adjust the permittivity and permeability independently. Additionally, by tuning the electric and magnetic resonances, an MTM can be impedance-matched 


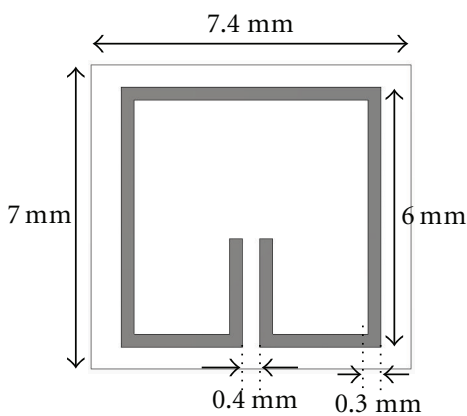

(a)

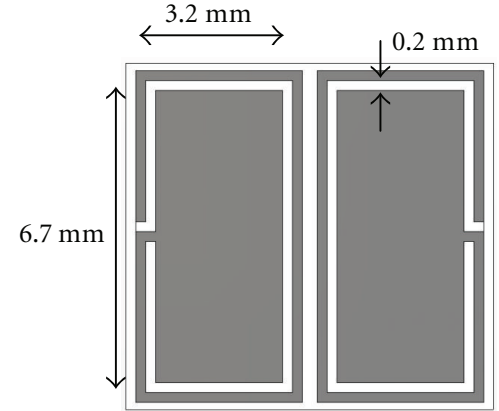

(b)

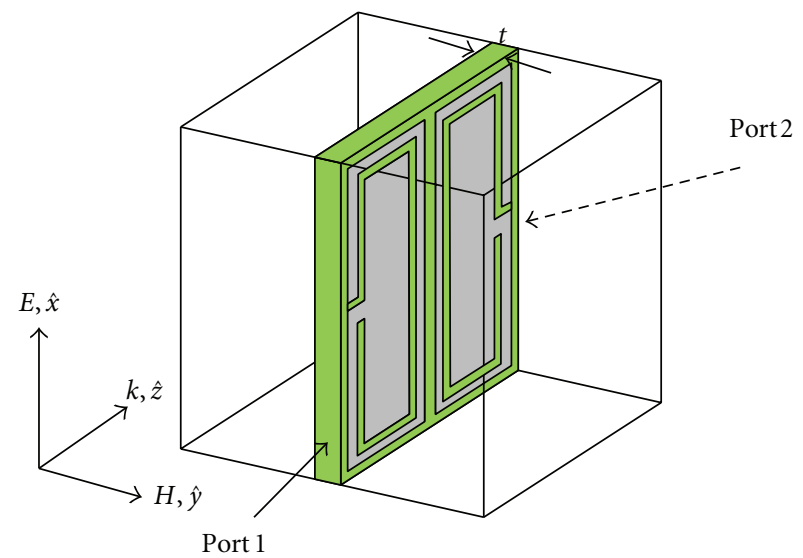

(c)

FIGURE 5: Geometry of the proposed MTM absorber cell: (a) top view (SRR); (b) bottom view (OCSRRs); (c) single unit cell showing the propagation direction of incident electromagnetic wave.

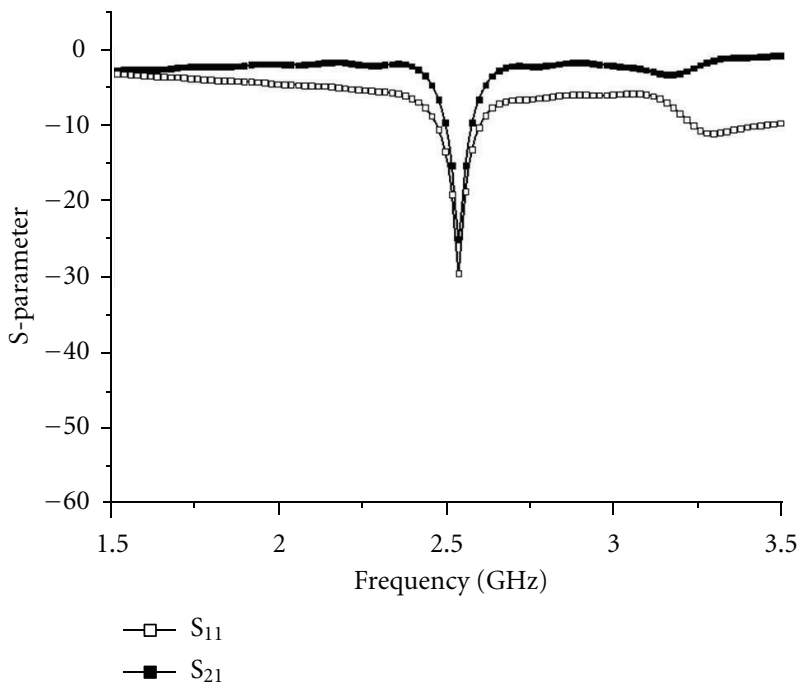

FIGURE 6: Simulated S-parameters for the proposed MTM absorber cell.

to free space. As a result, $100 \%$ absorbance is theoretically possible. The geometry of the proposed MTM absorber unit cell is shown in Figure 5. Two OCSRRs and a SRR structure are placed on both sides of the FR-4 dielectric substrate (relative permittivity $=4.6$, thickness $=2 \mathrm{~mm}$ ). Two OCSRRs are located with even symmetry on one side

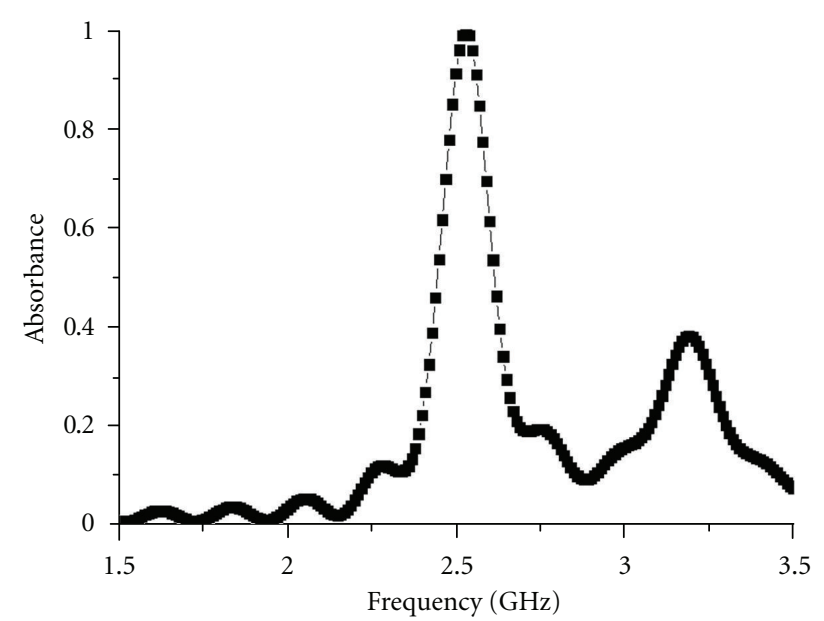

FIGURE 7: Calculated absorbance for proposed MTM absorber cell.

of the FR-4 substrate. We constructed an MTM unit cell using an OCSRR and SRR arrangement. The OCSRR is derived from the two former planar resonant structures: the open split ring resonator (OSRR) and CSRR. As compared to the SRR and CSRR, the OCSRR has the half resonant frequency with identical dimensions; it can be modeled as an open parallel resonant circuit. The OCSRR uses a modified CSRR structure exhibiting a negative permittivity; the SRR structure exhibits a negative permeability. The 


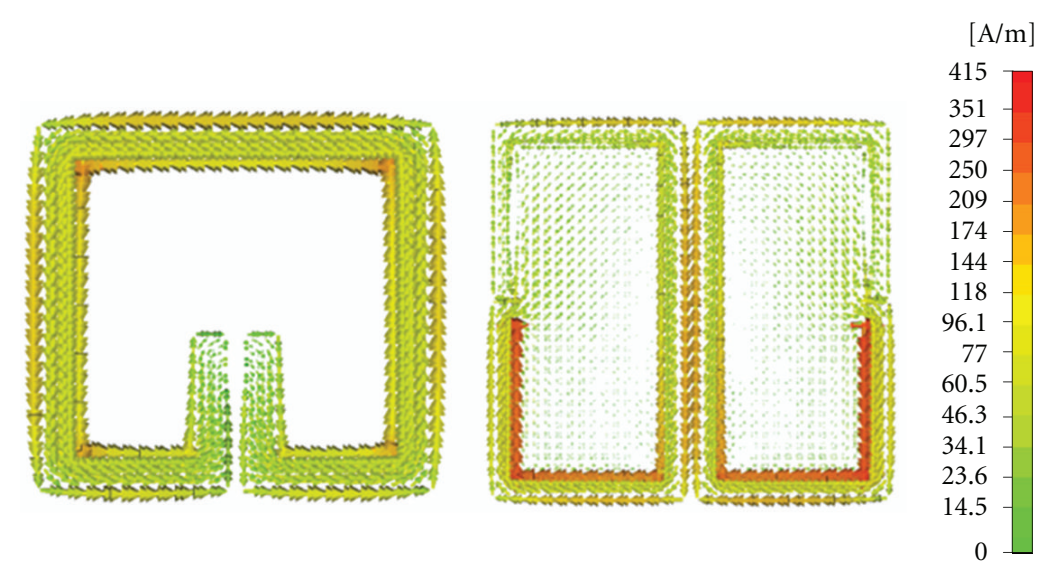

(a)
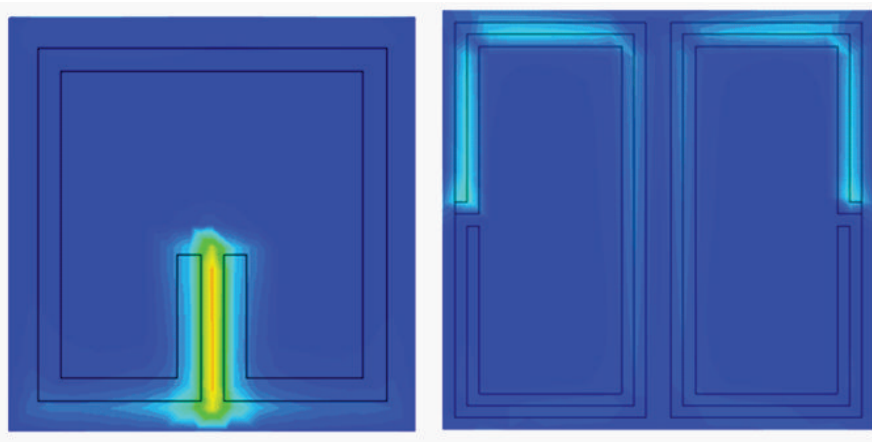

$\left[\mathrm{W} / \mathrm{m}^{3}\right]$

$1.30 \mathrm{e} 7$

$1.16 \mathrm{e} 7$

$1.06 \mathrm{e} 7$

$9.59 \mathrm{e} 6$

$8.63 \mathrm{e} 6$

$7.71 \mathrm{e} 6$

$6.83 \mathrm{e} 6$

$5.99 \mathrm{e} 6$

$4.42 \mathrm{e} 6$

$3.68 \mathrm{e} 6$

$2.97 \mathrm{e} 6$

$2.29 \mathrm{e} 6$

$1.64 \mathrm{e} 6$

$1.02 \mathrm{e} 6$

$4.29 \mathrm{e} 5$

(b)

FIGURE 8: Simulated results: (a) surface current densities; (b) average power loss densities in absorber plane.

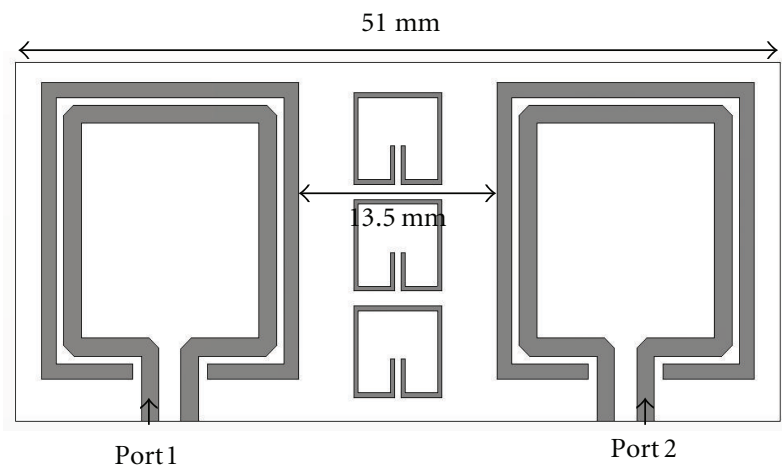

(a)

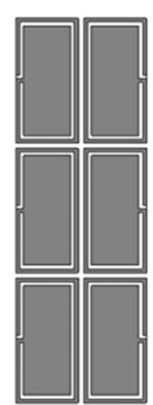

(b)

FIGURE 9: Geometry of the proposed antenna with three cells: (a) top view; (b) bottom view.

proposed absorber unit cell based on the double negative MTM structure is shown to reach a thickness on the order of $0.059 \lambda_{0}$ at the frequency of $2.54 \mathrm{GHz}$. The frequency characteristics of the unit cell structure are simulated by using a periodic boundary condition $(\mathrm{PBC})$ method; the dimensions of the unit cell are $7.4 \mathrm{~mm} \times 7 \mathrm{~mm} \times 2 \mathrm{~mm}$.

The proposed unit cell is placed inside a waveguide with PBC walls and a vertically polarized TEM wave impinged upon this structure from input port 1 . The scattering parameters of this MTM unit cell are then simulated, and the absorbance is calculated using the equation: $A=1-\left|S_{11}\right|^{2}-$ $\left|S_{21}\right|^{2}$. The simulated scattering parameters for the proposed unit cell are plotted in Figure 6. We observe that both the magnitudes of the reflection and transmission coefficients are very low at the resonance frequency of $2.54 \mathrm{GHz}$, which indicates strong absorption of the EM wave energy. The calculated absorbance curve using the simulated S-parameter in Figure 6 is plotted in Figure 7. The maximum absorbance 


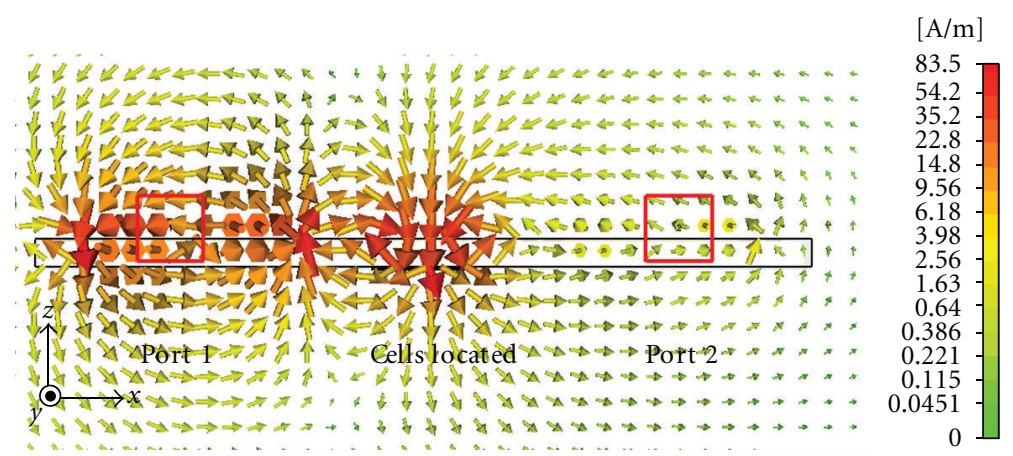

(a)

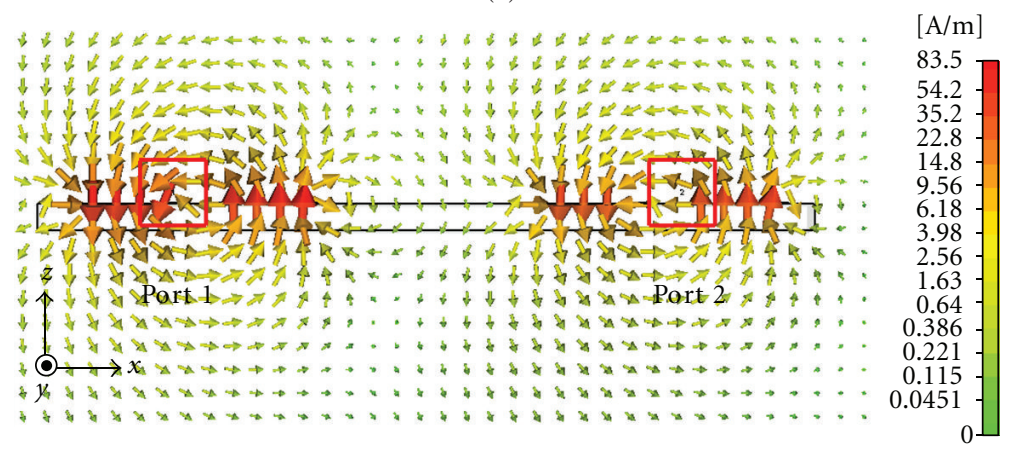

(b)

FIGURE 10: Comparison of the simulated magnetic field distributions of the antenna when a signal was fed to port $1(f=2.54 \mathrm{GHz})$ : (a) with MTM absorber cells; (b) without MTM absorber cells.

peak is $98 \%$, and it exhibits $12 \%$ of full-width half maximum (FWHM) bandwidth at $2.54 \mathrm{GHz}$.

As shown in Figure 8(a), the countercirculating current flow on the SRR provides magnetic resonance, and stronger current density occurs in the shortened end of the OCSRRs, which provides electric resonance at the resonance frequency of $2.54 \mathrm{GHz}$. The distribution of the average power loss density in the absorber plane at the resonance frequency is shown in Figure $8(\mathrm{~b})$. The power loss is concentrated strongly in the gaps of the SRR, which is more extensive than the gap of the OCSRRs. For the resonance at $2.54 \mathrm{GHz}$, the power loss has mainly taken place in the space neighboring the gap of the SRR. We should note that the absorption arises from the dielectric loss of the spacer material due to the concentrated electromagnetic wave in the gap locations.

\section{The Loop Antennas with Absorber Cells}

When a time-varying magnetic field, polarized normal to the plane of the SRR, is incident upon it, circulating currents are induced within the ring metallic patterns, resulting in a magnetic response. However, if the EM waves are incident normal to the planar SRR structure with the magnetic field lying in the plane of the SRRs, this does not excite a magnetic response. The geometry of the two proposed loop antennas with the three MTM absorber cells is shown in Figure 9. It consists of two loop antennas and three MTM absorber cells; the total antenna size was $51 \mathrm{~mm} \times 23.6 \mathrm{~mm} \times 2 \mathrm{~mm}$. In order to excite a magnetic response for the SRR, the
MTM absorbers are placed parallel to the loop antennas so that the magnetic field lies completely perpendicular to the plane of the SRR, as shown in Figure 9(a). The interelement spacing between two loop antennas is set to $13.5 \mathrm{~mm}\left(0.23 \lambda_{0}\right.$ at $2.54 \mathrm{GHz}$ ). The comparison of the simulated magnetic field distributions for the antenna with/without the MTM absorber cells is shown in Figure 10. When a signal with a frequency of $2.54 \mathrm{GHz}$ is applied to port 1 and the MTM absorber cells are not used, most of the magnetic fields are induced onto the two vertical loop's arms near port 1 and some parts of the radiated field from the loop antenna located at port 1 are coupled to the loop antenna located at port 2, as shown in Figure 10(b). When the MTM absorber cells are placed in the orthogonal direction against the magnetic fields, most of the magnetic fields are not coupled to the loop antenna at port 2, as shown in Figure 10(a). As a result, the port-to-port isolation between the two loop antennas is reduced by using the MTM absorber cells between the two antennas.

A comparison of the simulated radiation patterns for the proposed antennas with/without absorber cells at the frequency of $2.45 \mathrm{GHz}$ are shown in Figure 11. The proposed loop antenna in this paper has a symmetric loop pattern with an unbalanced feeding scheme. When the loop antenna is fed with an unbalanced feed, both even mode and odd mode can be simultaneously excited. As shown in Figure 3(a), the current distribution on the loop strip is equal and in same direction. This behavior implies that the loop antenna excites the even mode at $2.54 \mathrm{GHz}$. As a result, this design gives rise to near omnidirectional azimuth radiation pattern instead of 


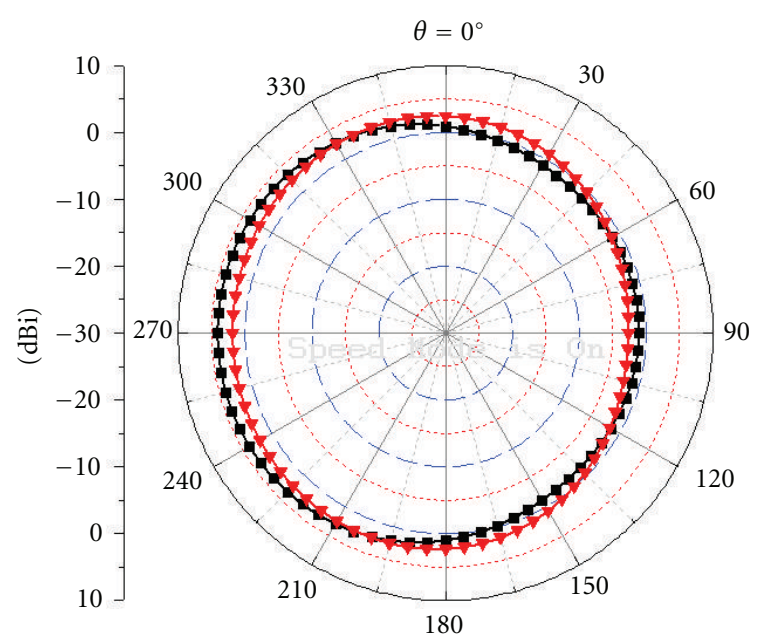

(a)

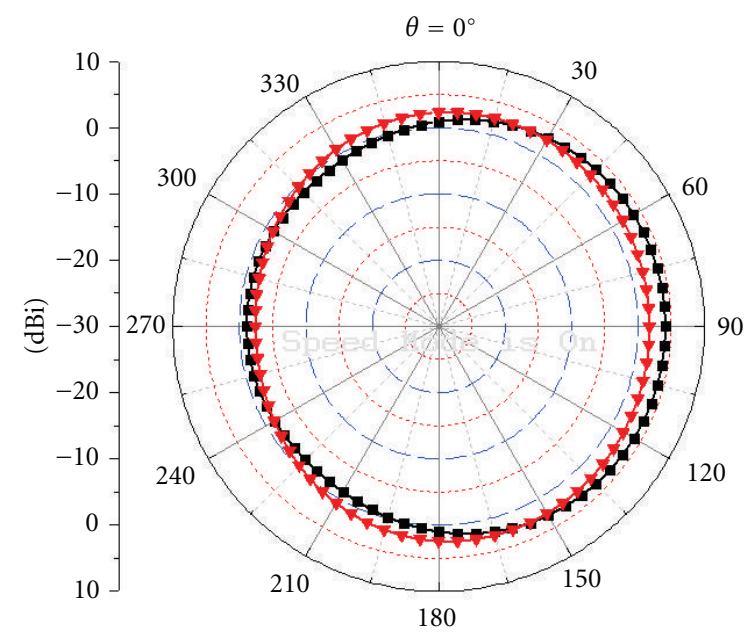

- Without cell

$\rightarrow$ With cell

(c)

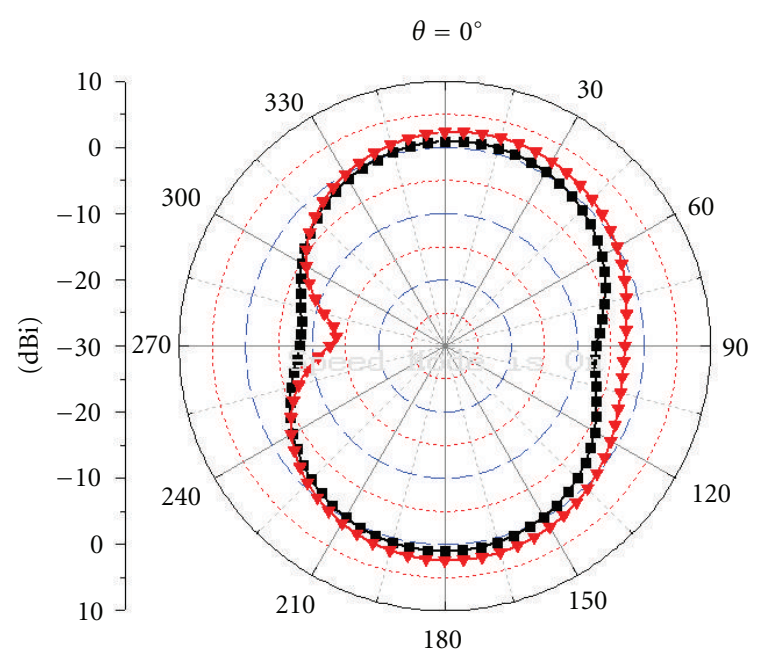

(b)

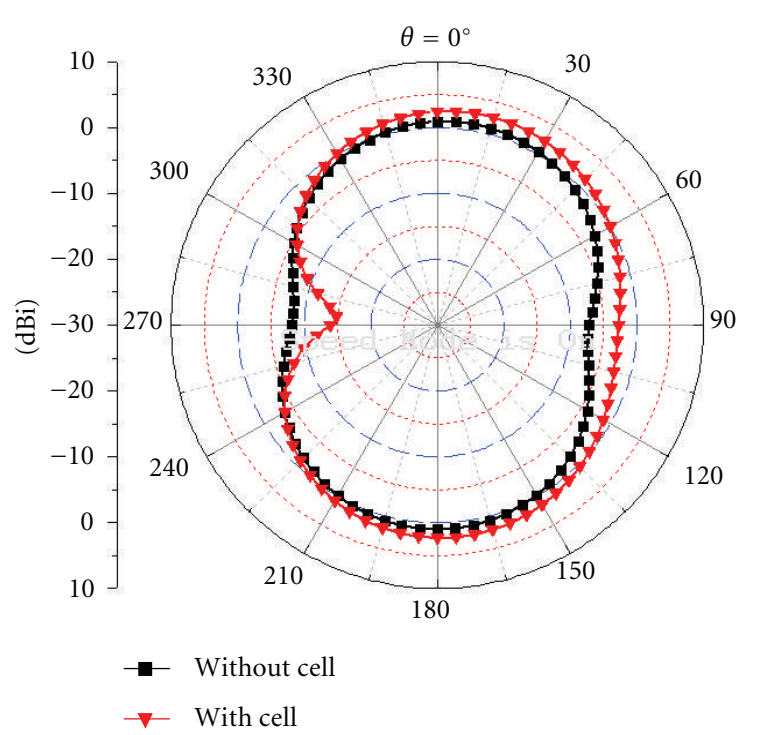

(d)

FIGURE 11: Comparison of the simulated radiation pattern at $2.54 \mathrm{GHz}$ : (a) $x$-z plane (antenna 1); (b) $y-z$ plane (antenna 1); (c) $x$ - $z$ plane (antenna 2); (d) $y-z$ plane (antenna 2).

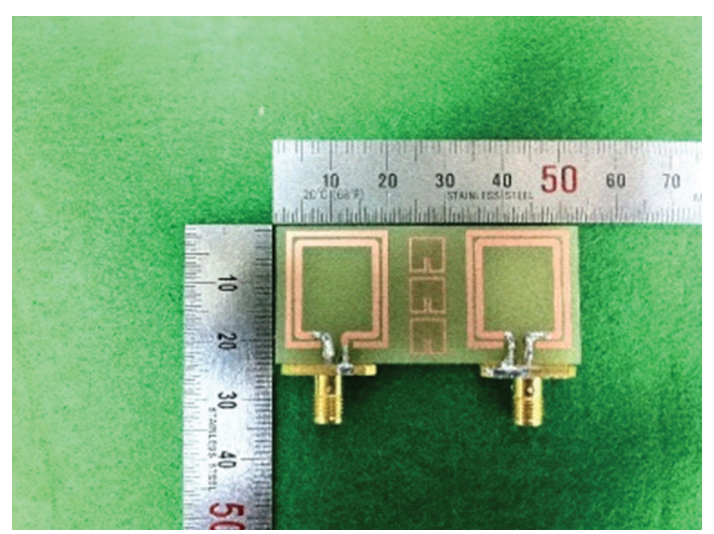

(a)

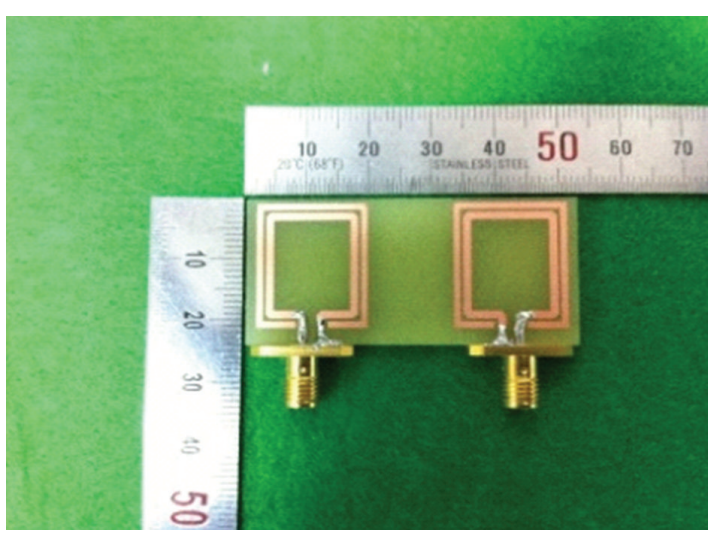

(b)

FIgURE 12: Photographs of the fabricated antenna (top view): (a) antenna with cells; (b) antenna without cells. 


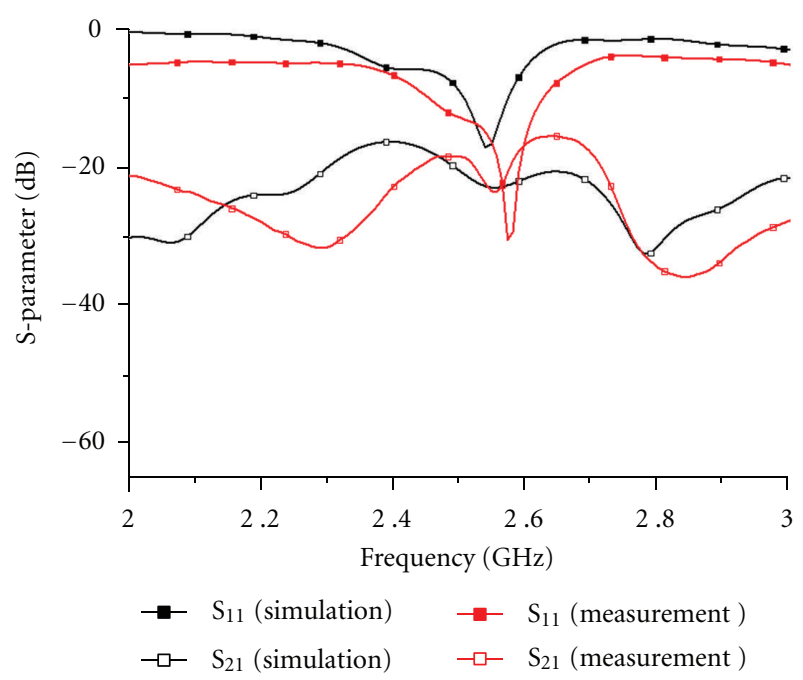

Figure 13: Comparison of the simulated and measured Sparameters for the proposed loop antenna with cells.

the figure-of-eight radiation pattern typical of a small loop antenna. The radiation patterns of the antenna without cells are near omnidirectional in the elevation plane $(x-z$ plane and $y-z$ plane). But, the power level of the antenna with cells is reduced at $\theta=90^{\circ}$ and $270^{\circ}$ due to the absorber cells.

\section{The Fabricated Antenna Measurement Results}

In order to verify the simulations, two loop antennas with three double negative MTM absorber cells are fabricated on a FR- 4 substrate with the relative dielectric constant 4.6 and a thickness of $2 \mathrm{~mm}$; two $50 \Omega$ SMA connecters are installed. The photographs of the two prototype fabricated antennas are shown in Figure 12. Comparisons of the simulated and measured S-parameter results for the proposed antennas with absorber cell are shown in Figure 13. The measured Sparameters are similar to the simulated results. When three absorber cells are placed between the antennas, the isolation $\left|S_{21}\right|$ shows a $10 \mathrm{~dB}$ improvement compared to the isolation of the antennas without the absorber cells. A maximum isolation of $24 \mathrm{~dB}$ is achieved at $2.54 \mathrm{GHz}$. The operating bandwidth of the proposed antenna is slightly lower due to the absorber cells between the two loop antennas. The comparison of the measured gain radiation patterns for the two prototype antennas with/without absorber cells is presented in Figure 14. In the measurement, the antenna placed in left side (antenna 1) in Figure 12 is fed and the antenna placed in right side (antenna 2) is terminated with a matched load. The measured radiation patterns of the prototypes are not very different compared to the simulated radiation patterns. These antennas exhibit an almost omnidirectional radiation pattern. However, some differences can be observed due to the absorber cells. As compared with measured radiation patterns at the elevation plane, the antenna gains near $\theta=$ $90^{\circ}$ (in the $x z$-plane) and $260^{\circ}$ (in the $y z$-plane) are slightly reduced when the absorber cells are used. A comparison

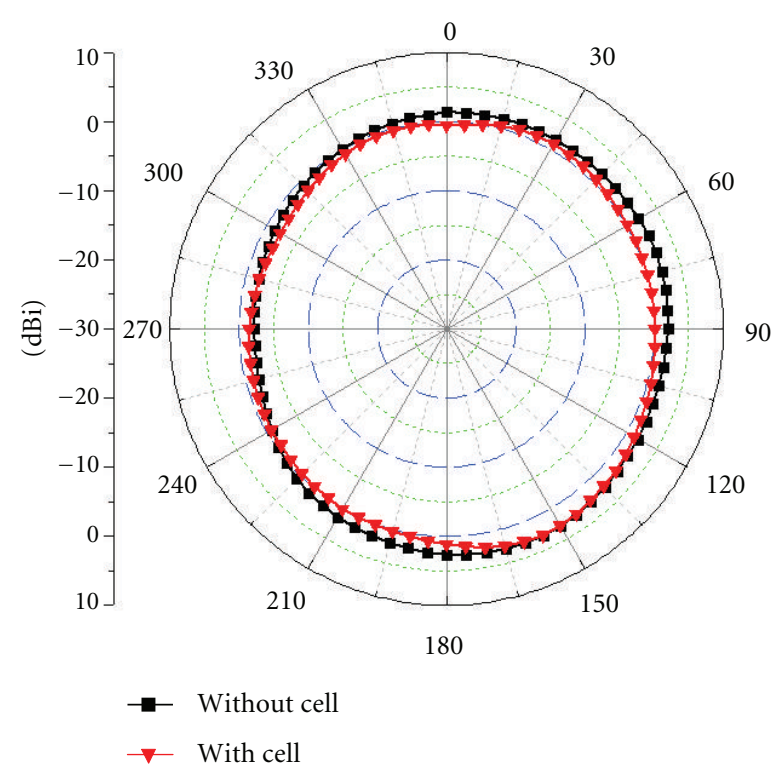

(a)

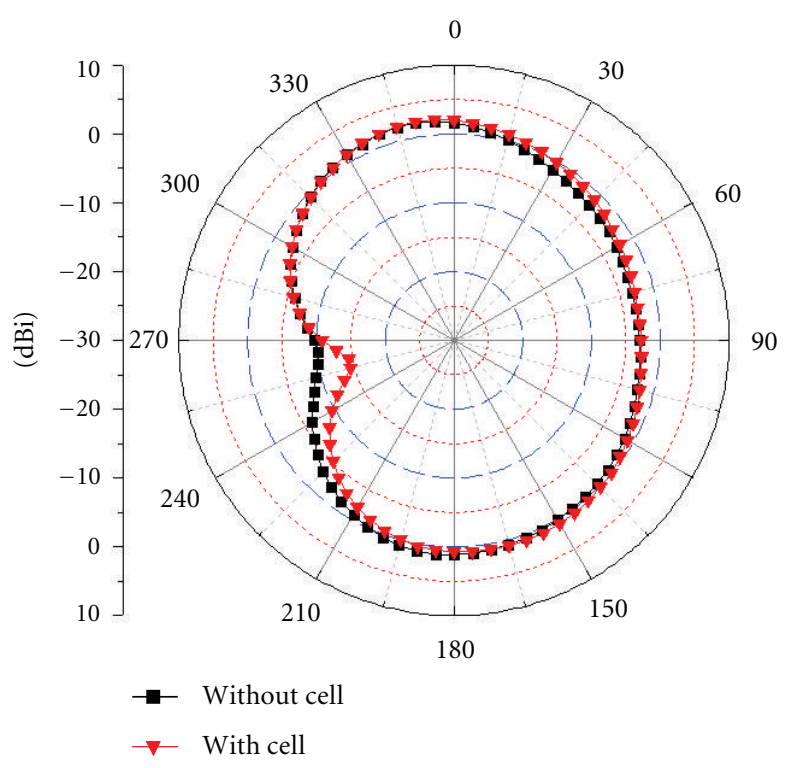

(b)

Figure 14: Comparison of the measured radiation patterns with/ without cells at $2.54 \mathrm{GHz}$ : (a) $x z$-plane; (b) $y z$-plane.

of the simulated and measured magnitude of the isolation level and efficiency for the proposed antennas with/without cells at the frequency of $2.54 \mathrm{GHz}$ are listed in Table 1 . The measured efficiency of the proposed antenna was calculated using the measured S-parameters. The measured efficiency of the proposed antenna with/without cells was $75 \%$ and $83 \%$, respectively.

\section{Conclusion}

This paper presents a new method used for improving the isolation between two closely spaced small loop antennas without a ground plane. The proposed antenna structures 
TABLE 1: Comparison of the simulated and measured performances for the proposed loop antenna.

\begin{tabular}{lcc}
\hline & $\begin{array}{c}\text { Antenna without } \\
\text { absorber cells }\end{array}$ & $\begin{array}{c}\text { Antenna with } \\
\text { absorber cells }\end{array}$ \\
\hline Simulated isolation $(\mathrm{dB})$ & 12 & 23 \\
Simulated efficiency (\%) & 83 & 80 \\
Measured isolation (dB) & 14 & 24 \\
Measured efficiency (\%) & 83 & 75 \\
\hline
\end{tabular}

are composed of two small loop antennas and three double negative MTM absorber unit cells exhibiting a negative permittivity and permeability at the tuned frequency of $2.54 \mathrm{GHz}$. The proposed absorber cell consists of a doublelayered structure employing a SRR and two OCSRRs. They are put on top of each other and have been shown to effectively absorb most of the impinging power. Both of them have same resonance frequency of $2.54 \mathrm{GHz}$. The proposed absorber cells can be effectively used in order to obtain a high isolation between the two closely spaced antennas. The two prototype antennas that occupy $51 \mathrm{~mm} \times 23.6 \mathrm{~mm} \times 2 \mathrm{~mm}$ were fabricated and measured. By placing the absorber cells in the middle of two loop antennas, we achieved a $10 \mathrm{~dB}$ port-to-port isolation improvement which led to a measured total efficiency of over 75\%. Although the efficiency of the antenna is slightly decreased due to the inserted absorber cells, this technique can be applied to improve the isolation in multiantenna systems.

\section{Acknowledgments}

This research was supported by Basic Science Research Program through National Research Foundation of Korea (NRF) funded by the Ministry of Education, Science and Technology (no. 2010-0011646) and Kyonggi University Research Assistant Fellowship 2011.

\section{References}

[1] M. A. Jensen and J. W. Wallace, "A review of antennas and propagation for MIMO wireless communications," IEEE Transactions on Antennas and Propagation, vol. 52, no. 11, pp. 2810 2824, 2004.

[2] C. Y. Chiu, C. H. Cheng, R. D. Murch, and C. R. Rowell, "Reduction of mutual coupling between closely-packed antenna elements," IEEE Transactions on Antennas and Propagation, vol. 55, no. 6, pp. 1732-1738, 2007.

[3] Y. S. Shin and S. O. Park, "Spatial diversity antenna for WLAN application," Microwave and Optical Technology Letters, vol. 49, no. 6, pp. 1290-1294, 2007.

[4] A. Diallo, C. Luxey, P. Le Thuc, R. Staraj, and G. Kossiavas, "Enhanced two-antenna structures for universal mobile telecommunications system diversity terminals," IET Microwaves, Antennas and Propagation, vol. 2, no. 1, pp. 93-101, 2008.

[5] A. Chebihi, C. Luxey, A. Diallo, P. Le Thuc, and R. Staraj, "A novel isolation technique for closely spaced PIFAs for UMTS mobile phones," IEEE Antennas and Wireless Propagation Letters, vol. 7, pp. 665-668, 2008.
[6] N. Engheta and R. W. Ziolkowski, Metamaterials: Physics and Engineering Explorations, Wiley-Intersciencce, Hoboken, NJ, USA, 2006.

[7] N. I. Landy, S. Sajuyigbe, J. J. Mock, D. R. Smith, and W. J. Padilla, "Perfect metamaterial absorber," Physical Review Letters, vol. 100, no. 20, Article ID 207402, 2008.

[8] A. Velez, F. Aznar, J. Bonache, J. M. Velazquez-Ahumada, and F. Martin, "Open complimentary split ring resonators (OCSRRs) and their application to wideband CPW band pass filters," IEEE Microwave Theory and Wireless Component Letters, vol. 19, pp. 197-199, 2009. 

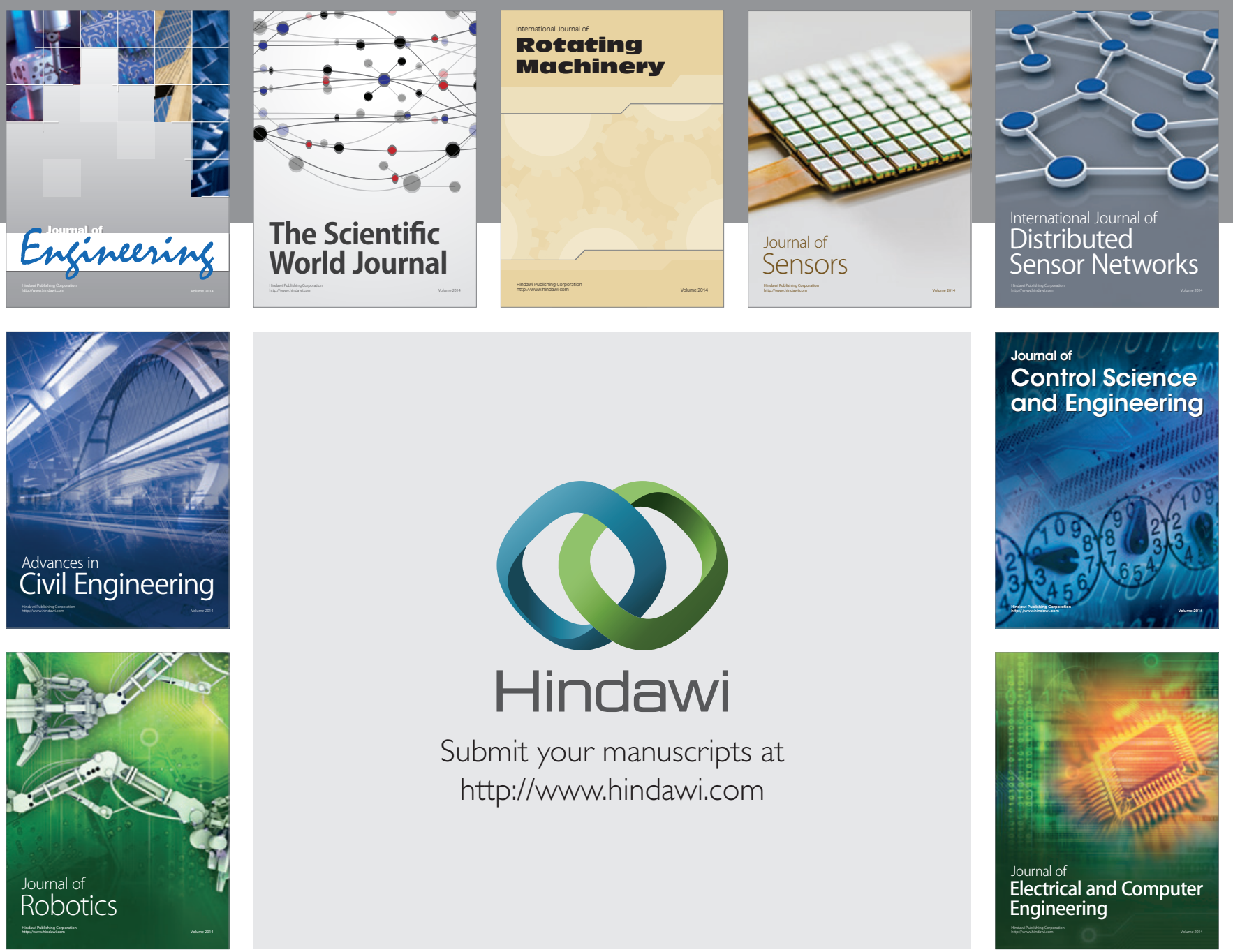

Submit your manuscripts at

http://www.hindawi.com
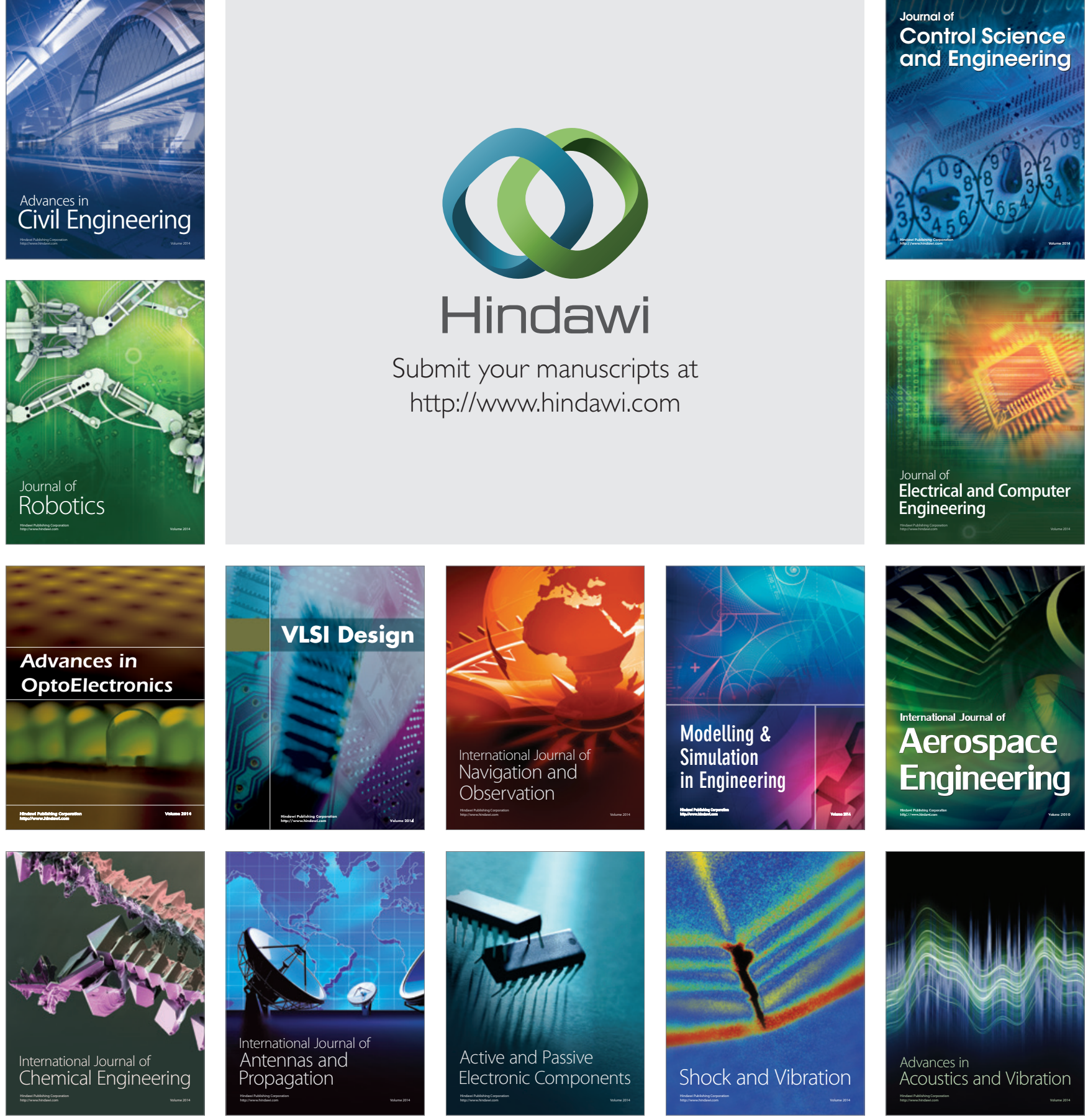